\title{
Reproducibility of volumetric intravascular ultrasound radiofrequency-based analysis of coronary plaque composition in vivo
}

\author{
Marc Hartmann · Eline S. K. Mattern · Jennifer Huisman · Gert K. van Houwelingen • \\ Frits H. A. F. de Man · Martin G. Stoel · Peter W. Danse · Hans W. Louwerenburg • \\ Clemens von Birgelen
}

Received: 12 March 2008/Accepted: 30 June 2008/Published online: 13 August 2008

(C) Springer Science+Business Media, B.V. 2008

\begin{abstract}
Intravascular ultrasound radiofrequency (RF-IVUS) data permit the analysis of coronary plaque composition in vivo and is used as an endpoint of ongoing pharmacological intervention trials. We assessed the reproducibility of volumetric RF-IVUS analyses in mild-to-moderately diseased atherosclerotic human coronary arteries in vivo. A total of 9,212 IVUS analyses on cross-sectional IVUS frames was performed to evaluate the reproducibility of volumetric RF-IVUS measurements in 33 coronary segments with a length of $27 \pm 7 \mathrm{~mm}$. For vessel, lumen, and plaque + media volume the relative measurement differences $(P=\mathrm{NS}$ for all $)$ were $(\mathrm{A}=$ intraobserver comparison, same pullback) $-0.40 \pm 1.0 \% ;-0.48 \pm$ $1.4 \% ;-0.35 \pm 1.6 \%,(\mathrm{~B}=$ intraobserver comparison,
\end{abstract}

M. Hartmann - E. S. K. Mattern - J. Huisman .

G. K. van Houwelingen - F. H. A. F. de Man .

M. G. Stoel - P. W. Danse · H. W. Louwerenburg .

C. von Birgelen $(\square)$

Department of Cardiology, Thoraxcentrum Twente,

Medisch Spectrum Twente, Haaksbergerstraat 55,

Enschede 7513ER, The Netherlands

e-mail: c.vonbirgelen@ziekenhuis-mst.nl

C. von Birgelen

Institute for Biomedical Technology (BMTI),

University of Twente, Enschede,

The Netherlands repeated pullback) $-0.42 \pm 1.2 \% ;-0.52 \pm 1.8 \%$; $-0.43 \pm 4.5 \%(\mathrm{C}=$ interobserver comparison, same pullback) $0.71 \pm 1.8 \% ; 0.71 \pm 2.2 \%$, and $0.89 \pm$ $5.0 \%$, respectively. For fibrous, fibro-lipidic, calcium, and necrotic-core volumes the relative measurement differences $(P=\mathrm{NS}$ for all) were (A) $0.45 \pm 2.1 \%$; $-1.12 \pm 4.9 \% ;-0.84 \pm 2.1 \% ;-0.22 \pm 1.8 \%$, (B) $1.40 \pm 4.1 \% ; 1.26 \pm 6.7 \% ; 2.66 \pm 7.4 \% ; 0.85 \pm$ $4.4 \%$, and $(\mathrm{C})-1.60 \pm 4.9 \% ; 3.85 \pm 8.2 \% ; 1.66 \pm$ $7.5 \%$, and $-1.58 \pm 4.7 \%$, respectively. Of note, necrotic-core volume showed on average the lowest measurement variability. Thus, in mild-to-moderate atherosclerotic coronary artery disease the reproducibility of volumetric compositional RF-IVUS measurements from the same pullback is relatively high, but lower than the reproducibility of geometrical IVUS measurements. Measurements from repeated pullbacks and by different observers show acceptable reproducibilities; the volumetric measurement of the necrotic-core shows on average the highest reproducibility of the compositional RF-IVUS measurements

Keywords Coronary disease - Ultrasonics · Radiofrequency · Virtual histology
Abbreviations
IVUS Intravascular ultrasound
RF Radiofrequency
TCFA Thin-cap fibroatheroma
VH Virtual histology 


\section{Introduction}

Both, the progression of atherosclerotic plaque volume and unfavorable plaque tissue characteristics contribute to the overall risk of cardiovascular events [1-5]. Previous progression-regression studies with intravascular ultrasound (IVUS) reported a certain beneficial effect of anti-atherosclerotic pharmacological therapies on the progression of plaque volume; however, this can only partly explain the clinical benefit observed [6-10]. While conventional greyscale IVUS permits accurate quantification of plaque and vessel dimensions, it has significant limitations in the assessment of plaque composition [6, 7, 11-15].

As a consequence, a spectral analysis of IVUS radiofrequency (RF-IVUS) data has been developed and is increasingly used in clinical research [16-22]. RF-IVUS quantifies coronary plaque components (e.g., the necrotic core) with a high predictive accuracy, as demonstrated in vitro and in vivo [23-25].

Volumetric RF-IVUS data may be interesting endpoints of IVUS studies that evaluate anti-atherosclerotic pharmacological therapies [26]. However, there is a lack of information on the reproducibility of this approach [17]. Therefore, we assessed in atherosclerotic human coronary segments the reproducibility of volumetric RF-IVUS in vivo by comparing analyses from the same pullback, repeated pullbacks, and by different observers.

\section{Methods}

Study population

To assess the reproducibility of segmental IVUS-RF data analysis in mild-to-moderately diseased atherosclerotic human coronary arteries in vivo, we repeatedly analyzed IVUS data sets of 33 at least $20 \mathrm{~mm}$-long coronary segments from non-target and non-treated coronary arteries with an angiographic lumen diameter stenosis $<50 \%$ and absence of severe calcification or vessel tortuosity. Two motorized IVUS pullbacks at a speed of $0.5 \mathrm{~mm} / \mathrm{s}$ were available from 16 patients in regular sinus rhythm in whom clinicallydriven, elective diagnostic cardiac catheterizations $(\mathrm{n}=10)$ or percutaneous coronary interventions $(n=6)$ required IVUS assessment. Informed consent was obtained prior to cardiac catheterization from all patients in accordance with the directives of the Local Medical Ethics Committee.

\section{RF-IVUS}

Details regarding the RF-IVUS technique, also known as virtual histology IVUS, and validation have previously been described [16, 17, 23-25]. In brief, spectral analysis of IVUS radiofrequency data is used to create tissue maps in order to classify atherosclerotic plaques into four major components (fibrous, fibro-lipidic, calcium, and necrotic-core). Different plaque components were represented according to a dedicated color code (green, lightgreen, white and red) (Fig. 1).

\section{RF-IVUS acquisition}

RF-IVUS data was acquired with commercially available phased-array IVUS catheters (Eagle Eye Gold 2.9F $20 \mathrm{MHz}$, Volcano Corporation, Rancho Cordova, USA) by a dedicated RF-IVUS console (Volcano Therapeutics, Rancho Cordova, USA). The image acquisition was ECG-gated (Fig. 1). The catheter probe was advanced $\geq 10 \mathrm{~mm}$ distal to the most distal side-branch. Angiographic cine runs were performed to define the position of the IVUS catheter. After intracoronary injection of $200 \mu \mathrm{g}$ nitroglycerine, a continuous pullback of the IVUS catheter was performed using a motorized pullback device at $0.5 \mathrm{~mm} / \mathrm{s}$ (TrackBackII, Volcano Corporation, Rancho Cordova, USA). In the patients of the present study, a second pullback was performed using the same IVUS catheter. Data of both pullbacks were stored on hard disk.

\section{RF-IVUS analysis}

RF-IVUS analysis was performed off-line by two experienced IVUS analysts (M.H., E.S.K.M.). The first pullback (I) was divided into segments as the region of interest. Segments were located between two adjacent side-branches and contained no major calcification that could have limited quantitative assessment of vessel cross-sectional area. Subsequently, the same segment was identified in the second pullback (II) using side-by-side comparison of longitudinal and cross-sectional views. In fact, this approach was similar to the matching procedure as 

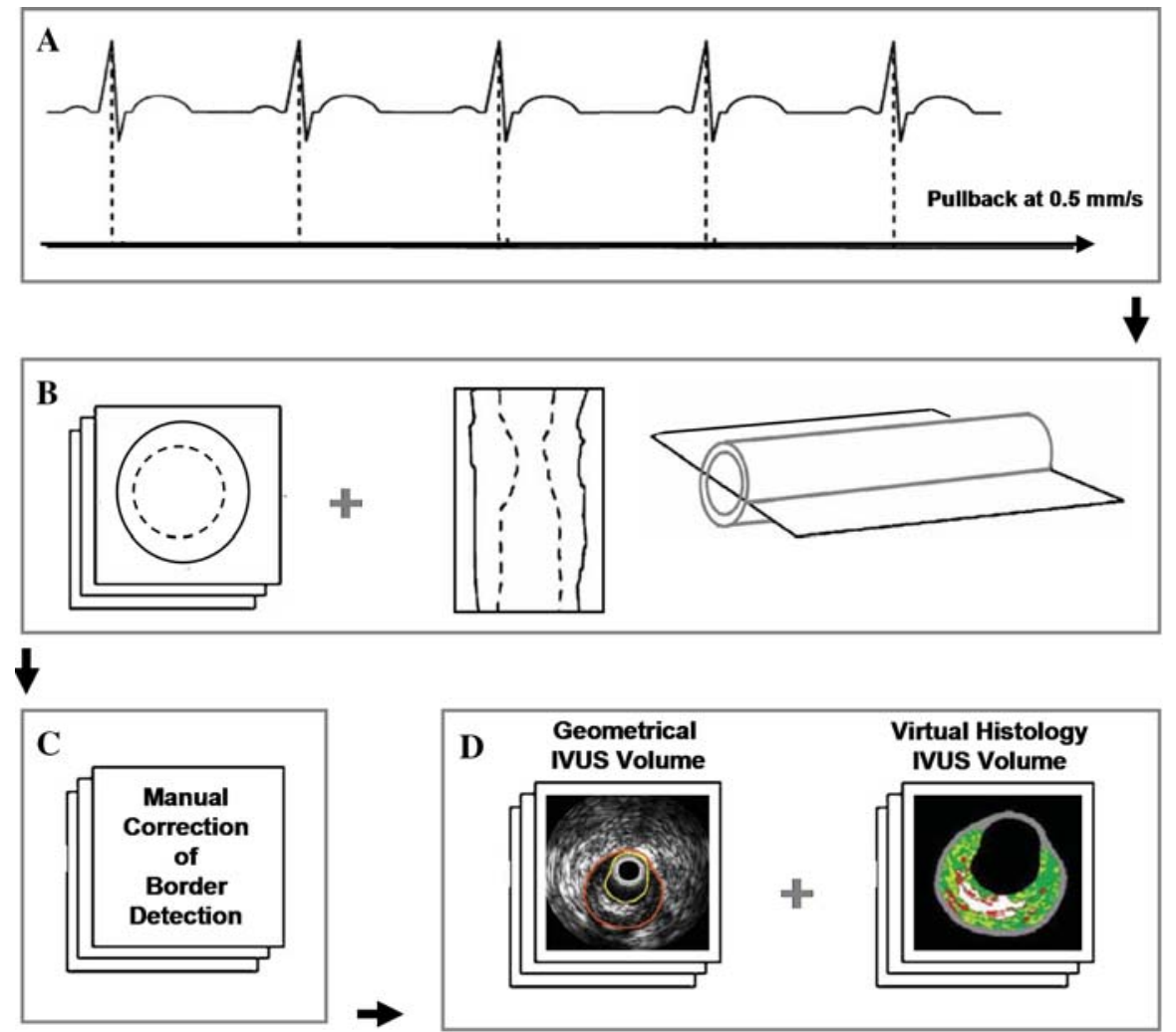

Virtual Histology IVUS Volume

Fig. 1 Image data acquisition and analysis. The R-wave peak of the ECG triggered image acquisition during a motorized pullback at a speed of $0.5 \mathrm{~mm} / \mathrm{s}$ (A). Automated contour detection of the luminal and vessel borders was performed on all IVUS images acquired. A longitudinal reconstruction of the segment was obtained from the entire three-dimensional "stack" of images (B). The direction of the longitudinal reconstruction was indicated by an arrowhead on the cross-

applied in serial IVUS studies [7-10]. The time required for the matching procedure was recorded. The vessel borders were traced using a semiautomatic contour detection program (IVUSLab 4.4, Volcano Corporation, Rancho Cordova, USA).

The borders of each cross-sectional image per segment were manually corrected if required to meet a high standard of accuracy. The lumen border was detected by tracing the leading edge of the intima, and the total vessel border by tracing the leading edge of the adventitia. Plaque + media (total vessel lumen) was used as a measure of atherosclerotic plaque $[11,12]$. The time required for the correction of the computerized contour detection was recorded. For each coronary segment, the vessel geometrics (lumen, total vessel and plaque + media volume) and sectional images. All contours were checked on the crosssectional frame and manual correction could be performed $(\mathbf{C})$. VH-IVUS analysis revealed (colour-encoded) information on plaque composition from the space between the lumen and vessel borders; volumetric data of the geometrical vessel dimension and of plaque composition were automatically generated for the segment (D)

the volumetric compositional RF-IVUS (fibrous, fibro-lipidic, calcium, and necrotic-core) were generated by applying Simpson's rule (Fig. 1) [22, 27]. Volumetric plaque burden (\%) was calculated as [(plaque volume/total vessel volume $) \times 100]$.

Each segment of the first pullback (I) was analyzed twice by the 1st observer (Ia and Ib) and once by the 2nd observer (Ic) after re-defining the segments of the first pullback; the corresponding segment of the second pullback (II) was analyzed once by the 1 st observer.

\section{Data analysis}

First, we compared repeated RF-IVUS measurements (volumetric vessel geometrics and composition) from 
the first pullback analyzed by the 1st observer (intraobserver comparison, Ia versus Ib). Second, we compared the RF-IVUS measurements from the first and second pullback analyzed by the 1st observer (intraobserver comparison, Ia versus II). Third, we compared RF-IVUS measurements from the first pullback between the 1st and 2nd observer (interobserver comparison, Ia versus Ic).

\section{Statistical analysis}

Analyses were performed with SPSS 13.0 (SPSS Inc., Chicago, Illinois). Dichotomous data are presented as frequencies. Quantitative data are presented as mean $\pm 1 \mathrm{SD}$ and compared using student t-test, linear regression analysis, or ANOVA for repeated measures with post hoc testing with Tukey honestly significant difference test. According to Bland and Altman, the agreement between two measurements were assessed by determining the mean $\pm 2 \mathrm{SD}$ of the between measurement differences [28]. A twosided $P$-value $<0.05$ was considered significant.

\section{Results}

Study population

Thirty-three atherosclerotic coronary segments (six proximal and six in the mid right, seven proximal and six in the mid left anterior descending, five proximal and three in the mid left circumflex coronary artery, respectively) from 16 patients with stable angina (11 men, age $58 \pm 8$ years) were included. The heart rate was $69 \pm 14 \mathrm{bpm}$. There were no complications related to the IVUS investigation.

RF-IVUS analysis and data

A total of 9,212 cross-sectional IVUS analyses were performed (70 \pm 21 frames/segment). Manual correction of the automatic border detection was required in $96 \pm 6 \%$ of frames/segment within $40 \pm 11 \mathrm{~min} /$ segment. Matching the segments of interest between the repeated pullbacks I and II required $13 \pm 5 \mathrm{~min} /$ segment.

The length of the segments was $27 \pm 7 \mathrm{~mm}$ (range: $20-46 \mathrm{~mm}$ ) with a volumetric plaque burden of $36 \pm 8 \%$ (range: $23-55 \%$ ). The atherosclerotic segments contained pre-dominantly fibrous (47\%) and necrotic-core $(25 \%)$ tissue. Further data are presented in Table 1.

Reproducibility of geometrical RF-IVUS (intraobserver and interobserver comparison)

Data on the agreement and variability of repeated geometrical RF-IVUS measurements (absolute and/or relative differences) showed a high reproducibility (Table 2 and Fig. 2). Repeated geometrical measurements were highly correlated for intraobserver comparison from the same pullback and repeated pullbacks, and for the interobserver comparison (r $\geq 0.999, \geq 0.997$, and $\geq 0.995 ; P<0.001$ for all; data not shown).

The limits of agreement (i.e., $2 \mathrm{SD}$ of mean difference), which indicate the expected relative difference between two measurements (Fig. 2), were higher for two observers who analyzed the same IVUS pullback than for the same observer who analyzed repeated pullbacks, with significantly lower relative differences for total vessel and lumen volume $(P<0.01$ for both, Table 2$)$.

Reproducibility of compositional RF-IVUS (intraobserver and interobserver comparison)

Data on the agreement and variability of repeated compositional measurements (absolute and/or relative differences) as presented in Table 2 and Figs. 3 and 4 showed an acceptable reproducibility. Repeated compositional measurements were highly correlated for intraobserver comparison from the same pullback and repeated pullbacks, and for the interobserver comparison $\quad(\mathrm{r} \geq 0.999, \geq 0.997, \quad$ and $\geq 0.995$; $P<0.001$ for all; data not shown). Of note, the interobserver comparison of compositional RF-IVUS measurements showed a higher variability than the intraobserver comparison from repeated IVUS pullbacks (Fig. 3) with significantly higher relative differences for fibrous and fibro-lipidic volume $(P<0.01$ for both, Table 2$)$.

Necrotic-core volume showed on average the lowest measurement variability of all plaque components in both, intraobserver and interobserver comparisons. 
Table 1 Volumetric RF-IVUS measurements of vessel geometrics and plaque composition of 1st and 2nd observer

\begin{tabular}{|c|c|c|c|c|}
\hline & \multicolumn{3}{|l|}{ 1st Observer } & \multirow{2}{*}{$\begin{array}{l}\text { 2nd Observer } \\
\text { Pullback Ic }\end{array}$} \\
\hline & Pullback Ia & Pullback Ib & Pullback II & \\
\hline \multicolumn{5}{|l|}{ Vessel geometrics } \\
\hline Total vessel volume $\left(\mathrm{mm}^{3}\right)$ & $458.98 \pm 118.1$ & $460.24 \pm 116.3$ & $460.85 \pm 118.1$ & $456.27 \pm 119.6$ \\
\hline Lumen volume $\left(\mathrm{mm}^{3}\right)$ & $290.05 \pm 77.7$ & $291.11 \pm 76.8$ & $291.33 \pm 77.5$ & $288.09 \pm 77.9$ \\
\hline Plaque + media volume $\left(\mathrm{mm}^{3}\right)$ & $168.93 \pm 70.4$ & $169.12 \pm 69.0$ & $169.32 \pm 70.3$ & $168.17 \pm 72.3$ \\
\hline Plaque burden $(\%)$ & $36.36 \pm 8.3$ & $36.33 \pm 8.2$ & $36.31 \pm 8.2$ & $36.34 \pm 8.5$ \\
\hline \multicolumn{5}{|l|}{ Plaque composition } \\
\hline Fibrous volume $\left(\mathrm{mm}^{3}\right)$ & $34.06 \pm 32.0$ & $34.10 \pm 32.0$ & $33.68 \pm 31.7$ & $34.35 \pm 32.1$ \\
\hline Fibrous $(\%)$ & $46.62 \pm 15.5$ & $46.44 \pm 15.8$ & $46.64 \pm 15.8$ & $46.98 \pm 15.3$ \\
\hline Fibro-lipidic volume $\left(\mathrm{mm}^{3}\right)$ & $5.66 \pm 7.7$ & $5.70 \pm 7.9$ & $5.55 \pm 7.5$ & $5.49 \pm 7.2$ \\
\hline Fibro-lipidic $(\%)$ & $6.94 \pm 4.8$ & $6.82 \pm 4.7$ & $6.93 \pm 4.6$ & $6.67 \pm 4.8$ \\
\hline Calcium volume $\left(\mathrm{mm}^{3}\right)$ & $12.46 \pm 9.5$ & $12.48 \pm 9.3$ & $12.36 \pm 9.5$ & $12.48 \pm 9.9$ \\
\hline Calcium (\%) & $21.85 \pm 11.7$ & $21.98 \pm 11.8$ & $21.73 \pm 11.8$ & $21.40 \pm 11.6$ \\
\hline Necrotic-core volume $\left(\mathrm{mm}^{3}\right)$ & $15.94 \pm 13.5$ & $16.00 \pm 13.5$ & $15.77 \pm 13.1$ & $16.15 \pm 13.3$ \\
\hline Necrotic-core $(\%)$ & $24.59 \pm 8.5$ & $24.62 \pm 8.7$ & $24.70 \pm 8.6$ & $24.83 \pm 8.7$ \\
\hline
\end{tabular}

\section{Discussion}

Why measure changes in plaque composition?

Serial IVUS has become an established imaging method to measure effects of cardiovascular risk factors and drugs on coronary plaque dimensions [610, 29-31]. Plaque progression as measured by IVUS may be linked to a significant increase in risk of clinical events as predicted by established risk-scores [31]. Therefore, IVUS derived surrogate endpoints as an alternative to clinical endpoints may expedite the process of drug development and testing [6]. However, there is a contradiction between the significant clinical benefit of certain pharmacological interventions (e.g., statins) and the relatively small effect on plaque volume, which may be explained by a stabilizing effect on plaque composition $[6,7,10$, 26, 32].

Intravascular ultrasound radiofrequency analysis

RF-IVUS is an imaging technique that permits the characterization and quantification of plaque components in vivo (16, 17, 23-25]. Non-serial studies suggested a potential of RF-IVUS to detect high-risk coronary plaques (i.e., thin-cap fibroatheroma) and offered new insights into coronary pathology [18-22].
Currently, large serial IVUS studies are on the way that assess with a volumetric RF-IVUS approach changes in plaque composition to analyze the effect of atherosclerosis-modifying drugs on this new surrogate endpoint [33].

Rationale for the present study and main findings

An important prerequisite for the use of RF-IVUS data as an endpoint in serial studies is the assessment of its reproducibility [17, 34]. Drug-induced effects on coronary arterial dimensions and plaque composition may remain relatively small [7-10]. To date there is a lack of volumetric reproducibility data simulating a serial RF-IVUS study design (e.g., repeated pullbacks) [17, 34].

Therefore, we assessed in the present study the reproducibility of repeated RF-IVUS analyses of vessel geometrics and plaque composition in 33 coronary segments. The relative intraobserver difference of geometrical and compositional volumetric analyses in repeated pullbacks was relatively low $(<0.6 \%$ for all geometric vessel volumes; $<1 \%$ for necrotic-core and $<3 \%$ for fibrous, fibro-lipidic, and calcific plaque components) and showed good limits of agreement. Of note, the interobserver comparison of RF-IVUS of geometrical and compositional measurements from the same pullback showed on average 
Table 2 Intra- and interobserver measurement differences of volumetric vessel geometrics and plaque composition

$\begin{array}{lllllll}\begin{array}{l}\text { Intraobserver } \\ \text { comparison }\end{array} & \begin{array}{l}\text { Intraobserver } \\ \text { comparison } \\ \text { (same pullback) }\end{array} & \begin{array}{l}\text { Interobserver } \\ \text { comparison } \\ \text { (repeated }\end{array} & \begin{array}{l}\text { ANOVA } P \\ \text { (same pullback) }\end{array} & & P 2 & P 3 \\ & \begin{array}{l}\text { pullback) } \\ \text { Ia versus II }\end{array} & \text { Ia versus Ic } & & & & \\ & & & & & \\ & & & & & \end{array}$

\begin{tabular}{|c|c|c|c|c|c|c|c|}
\hline \multicolumn{8}{|l|}{ Vessel geometrics } \\
\hline$\Delta$ Total vessel volume $\left(\mathrm{mm}^{3}\right)$ & $-1.25 \pm 3.8^{*}$ & $-1.67 \pm 4.9 *$ & $2.72 \pm 8.0^{*}$ & $<\mathbf{0 . 0 1}$ & NS & $<\mathbf{0 . 0 5}$ & $<\mathbf{0 . 0 1}$ \\
\hline$\% \Delta$ Total vessel volume $(\%)$ & $-0.40 \pm 1.0$ & $-0.42 \pm 1.2$ & $0.71 \pm 1.8$ & $<\mathbf{0 . 0 1}$ & NS & $<\mathbf{0 . 0 1}$ & $<\mathbf{0 . 0 1}$ \\
\hline$\Delta$ Lumen volume $\left(\mathrm{mm}^{3}\right)$ & $-1.06 \pm 3.5^{*}$ & $-1.28 \pm 5.1^{*}$ & $1.95 \pm 6.5^{*}$ & $<\mathbf{0 . 0 5}$ & NS & NS & $<\mathbf{0 . 0 5}$ \\
\hline$\% \Delta$ Lumen volume $(\%)$ & $-0.48 \pm 1.4$ & $-0.52 \pm 1.8$ & $0.71 \pm 2.2$ & $<\mathbf{0 . 0 1}$ & NS & $<\mathbf{0 . 0 5}$ & $<\mathbf{0 . 0 5}$ \\
\hline$\Delta$ Plaque + media volume $\left(\mathrm{mm}^{3}\right)$ & $-0.19 \pm 2.9^{*}$ & $-0.39 \pm 5.8 *$ & $0.76 \pm 7.5^{*}$ & 0.7 & - & - & - \\
\hline$\% \Delta$ Plaque + media volume $(\%)$ & $-0.35 \pm 1.6$ & $-0.43 \pm 4.5$ & $0.89 \pm 5.0$ & 0.3 & - & - & - \\
\hline$\Delta$ Plaque burden $(\%)$ & $0.03 \pm 0.5^{*}$ & $0.05 \pm 1.2^{*}$ & $0.02 \pm 1.4^{*}$ & 0.9 & - & - & - \\
\hline$\% \Delta$ Plaque burden $(\%)$ & $0.04 \pm 1.4$ & $0.01 \pm 3.7$ & $0.20 \pm 4.2$ & 0.9 & - & - & - \\
\hline \multicolumn{8}{|l|}{ Plaque composition } \\
\hline$\Delta$ Fibrous volume $\left(\mathrm{mm}^{3}\right)$ & $-0.04 \pm 0.7 *$ & $0.38 \pm 2.0^{*}$ & $-0.28 \pm 1.0^{*}$ & 0.1 & - & - & - \\
\hline$\% \Delta$ Fibrous volume $(\%)$ & $0.45 \pm 2.1$ & $1.40 \pm 4.1$ & $-1.60 \pm 4.9$ & $<\mathbf{0 . 0 1}$ & NS & NS & $<\mathbf{0 . 0 1}$ \\
\hline$\Delta$ Fibrous $(\%)$ & $0.18 \pm 0.6^{*}$ & $-0.02 \pm 1.2 *$ & $-0.37 \pm 1.5^{*}$ & 0.1 & - & - & - \\
\hline$\% \Delta$ Fibrous $(\%)$ & $0.75 \pm 1.7$ & $0.24 \pm 2.8$ & $-1.00 \pm 4.2$ & 0.1 & - & - & - \\
\hline$\Delta$ Fibro-lipidic volume $\left(\mathrm{mm}^{3}\right)$ & $-0.04 \pm 0.3^{*}$ & $0.12 \pm 0.5^{*}$ & $0.17 \pm 0.8^{*}$ & 0.6 & - & - & - \\
\hline$\% \Delta$ Fibro-lipidic volume $(\%)$ & $-1.12 \pm 4.9$ & $1.26 \pm 6.7$ & $3.85 \pm 8.2$ & $<\mathbf{0 . 0 1}$ & NS & $<\mathbf{0 . 0 1}$ & $<\mathbf{0 . 0 1}$ \\
\hline$\Delta$ Fibro-lipidic $(\%)$ & $0.11 \pm 0.8^{*}$ & $0.00 \pm 0.6^{*}$ & $0.27 \pm 0.9^{*}$ & 0.6 & - & - & - \\
\hline$\% \Delta$ Fibro-lipidic $(\%)$ & $0.27 \pm 8.2$ & $-0.07 \pm 5.8$ & $6.03 \pm 9.1$ & $<\mathbf{0 . 0 1}$ & NS & $<\mathbf{0 . 0 5}$ & $<\mathbf{0 . 0 1}$ \\
\hline$\Delta$ Calcium volume $\left(\mathrm{mm}^{3}\right)$ & $-0.01 \pm 0.3^{*}$ & $0.10 \pm 0.8^{*}$ & $-0.02 \pm 1.0 *$ & 0.6 & - & - & - \\
\hline$\% \Delta$ Calcium volume $(\%)$ & $-0.84 \pm 2.1$ & $2.66 \pm 7.4$ & $1.66 \pm 7.5$ & 0.1 & - & - & - \\
\hline$\Delta$ Calcium $(\%)$ & $-0.13 \pm 0.4^{*}$ & $0.12 \pm 1.3^{*}$ & $0.45 \pm 1.5 *$ & 0.1 & - & - & - \\
\hline$\% \Delta$ Calcium $(\%)$ & $-0.51 \pm 1.7$ & $1.52 \pm 6.7$ & $2.26 \pm 6.9$ & 0.1 & - & - & - \\
\hline$\Delta$ Necrotic-core volume $\left(\mathrm{mm}^{3}\right)$ & $-0.05 \pm 0.4^{*}$ & $0.18 \pm 0.8^{*}$ & $-0.21 \pm 0.8^{*}$ & 0.1 & - & - & - \\
\hline$\% \Delta$ Necrotic-core volume $(\%)$ & $-0.22 \pm 1.8$ & $0.85 \pm 4.4$ & $-1.58 \pm 4.7$ & 0.1 & - & - & - \\
\hline$\Delta$ Necrotic-core $(\%)$ & $-0.03 \pm 0.4^{*}$ & $-0.11 \pm 1.0^{*}$ & $-0.23 \pm 0.8^{*}$ & 0.9 & - & - & - \\
\hline$\% \Delta$ Necrotic-core $(\%)$ & $0.07 \pm 1.8$ & $-0.34 \pm 3.6$ & $-0.90 \pm 3.4$ & 0.4 & - & - & - \\
\hline
\end{tabular}

P1 = Ia versus Ib vs. Ia versus II; P2 = Ia versus Ib vs. Ia versus Ic; and P3 = Ia versus II vs. Ia versus Ic. *Paired t-test Ia versus Ib; Ia versus II; and Ia versus Ic $P=$ NS for all. $\Delta=$ difference

Significant $P$-values are represented in bold

a somewhat higher variability than the intraobserver comparison of repeated pullbacks.

\section{Reproducibility of IVUS measurements}

The reproducibility of two-dimensional RF-IVUS has been tested by Rodriguez-Granillo et al. who compared repeated pullbacks from 16 coronary lesions [34]. The relative intraobserver difference was $\leq 3 \%$ for the cross-sectional area measurements of vessel geometrics; in general compositional measurements were more variable than geometrical measurements. The relative intraobserver difference was $\leq 6 \%$ for necrotic-core area, and $8-13 \%$ for fibrous, fibro-lipidic, and calcium areas [34]. Nevertheless, a volumetric IVUS approach is likely to result in a higher reproducibility. Kawasaki et al. reported intra- and interobserver variabilities of volumetric plaque compositional measurements ( $<3.6 \%$ for both) from 20 lesions from the same IVUS pullback by using the Integrated Backscatter IVUS technique, which is based on a different mathematical method compared to RF-IVUS by Virtual Histology [17, 26]. 


\section{Intraobserver Comparison (same pullback)}

RELATIVE $\triangle$ IN TOTAL VESSEL VOLUME (\%)

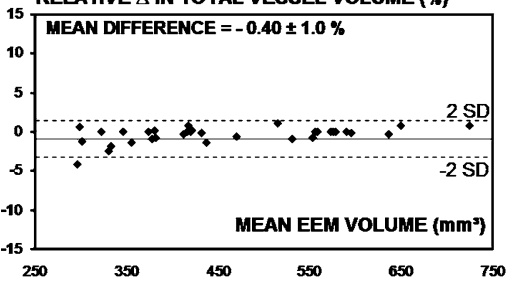

RELATIVE $\triangle$ IN LUMEN VOLUME (\%)

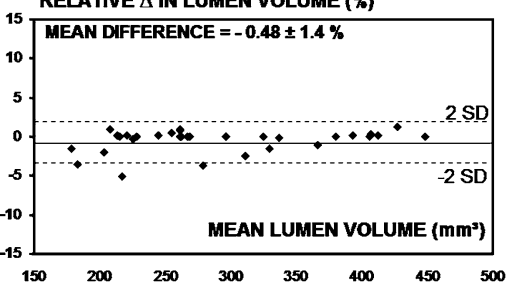

RELATIVE $\triangle$ IN PLAQUE+MEDIA VOLUME (\%)

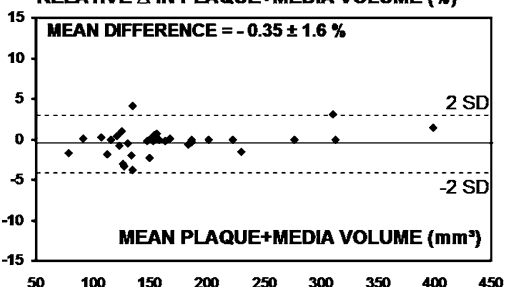

Intraobserver Comparison (repeated pullback)

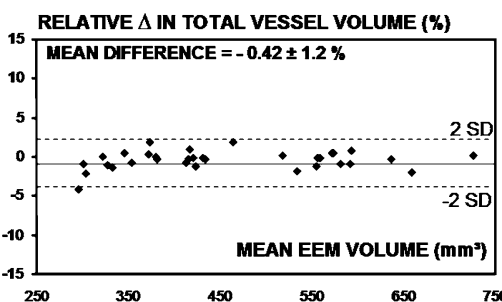

RELATIVE $\triangle$ IN LUMEN VOLUME (\%)

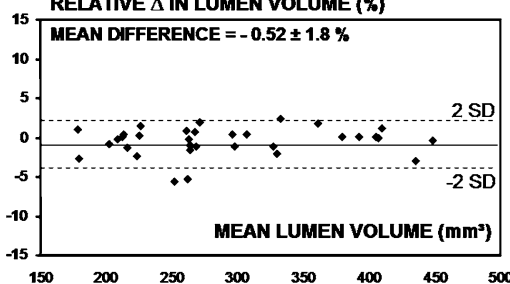

RELATIVE $\triangle$ IN PLAQUE+MEDIA VOLUME (\%)

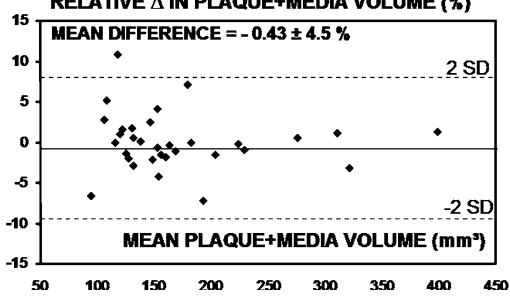

Interobserver Comparison (same pullback)

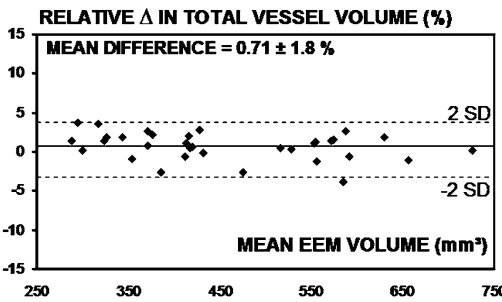

RELATIVE $\triangle$ IN LUMEN VOLUME (\%)

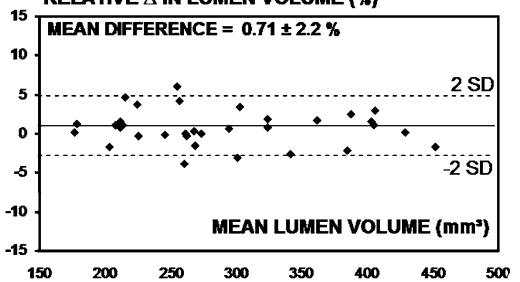

RELATIVE $\triangle$ IN PLAQUE+MEDIA VOLUME (\%)

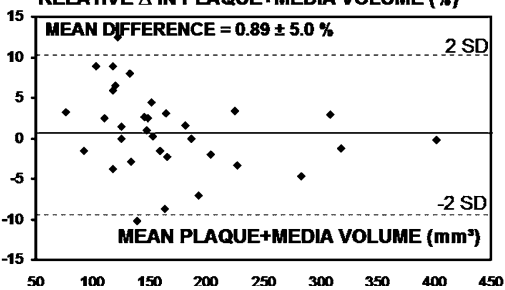

Fig. 2 Agreement of repeated VH-IVUS measurements of geometrical vessel volumes. Bland-Altman plots of intra- (left, mid) and interobserver (right) comparisons. $\Delta=$ difference

Variability of compositional RF-IVUS analyses is related to the variability of geometrical vessel analysis $[34,35]$. Tissue components mainly located in the center of an atherosclerotic plaque may be less sensitive to potential variation in border detection. Factors of variability in geometrical vessel analyses in a serial study design are-besides the quality of the basic IVUS images - the quality of matching the region of interest. Matching the cross-sectional target slice from baseline and follow-up examination is much more difficult with a two-dimensional $R F$-IVUS approach [11, 15]. The problem of matching has been significantly reduced by the use of volumetric IVUS, which visualizes an entire coronary segment, permits identification of one or more landmarks (e.g., adjacent side-branches), and is the current standard method in serial IVUS trials that address atherosclerosis-modifying strategies [7-10, 15].

In the present study, image acquisition was performed in an ECG-gated fashion. ECG-gated image acquisition prevents artifacts from cyclic movement of the IVUS catheter and the systolicdiastolic variation of the vessel dimensions which results in a higher reproducibility of geometrical IVUS measurements [13-15]. In agreement with our results, Okkels et al. demonstrated a high reproducibility of vessel geometrical measurements from repeated pullbacks using a dedicated ECG-gated volumetric grey-scale IVUS technique [15].

The necrotic-core

Necrotic-core tissue is the characteristic feature of socalled RF-IVUS derived thin-cap fibroatheroma (TCFA) [16-18]. Observational IVUS-RF studies demonstrated an association between RF-IVUS derived TCFA and both coronary and clinical instability [18, 19, 22]. RF-IVUS derived TCFA is generally defined as at least three consecutive twodimensional cross-sectional frames with $\geq 10 \%$ 


\section{Intraobserver Comparison \\ (same pullback)}
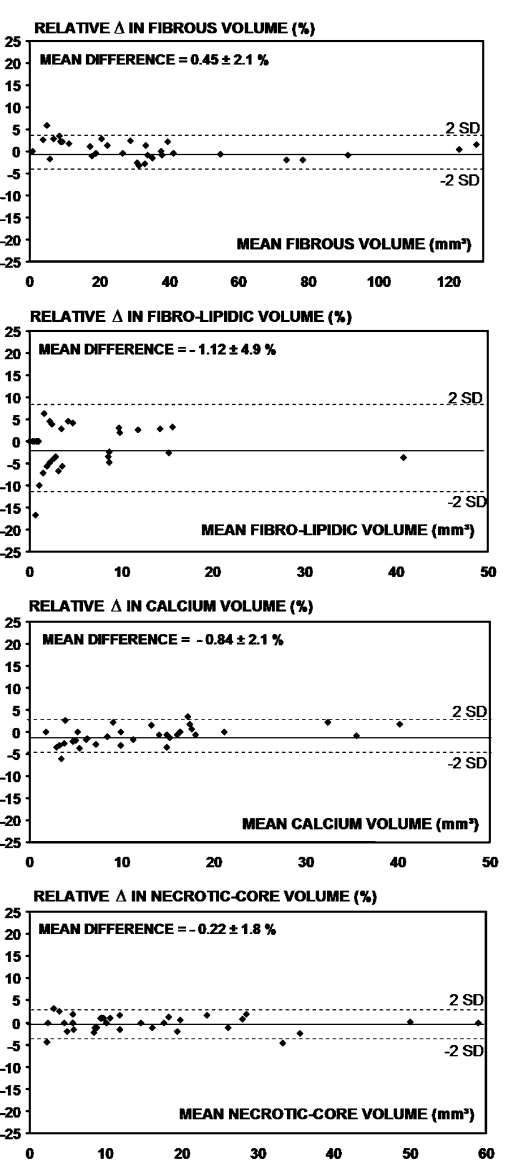

Intraobserver Comparison

(repeated pullback)

RELATIVE $\triangle$ IN FIBROUS VOLUME (\%)

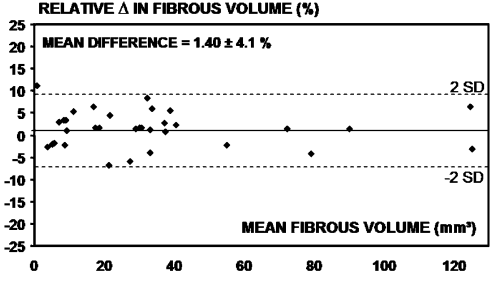

RELATIVE $\triangle$ IN FIBRO-LIPIDIC VOLUME (\%)

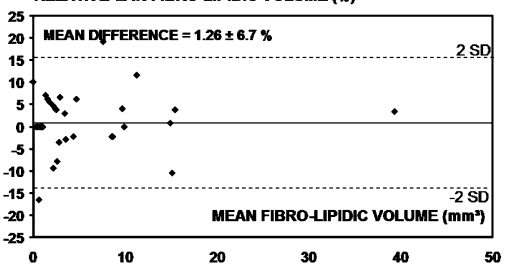

$\begin{array}{lcrr}0 & 10 & 20 & 30 \\ \text { RELATIVE } \Delta \text { IN CALCIUM VOLUME (\%) }\end{array}$
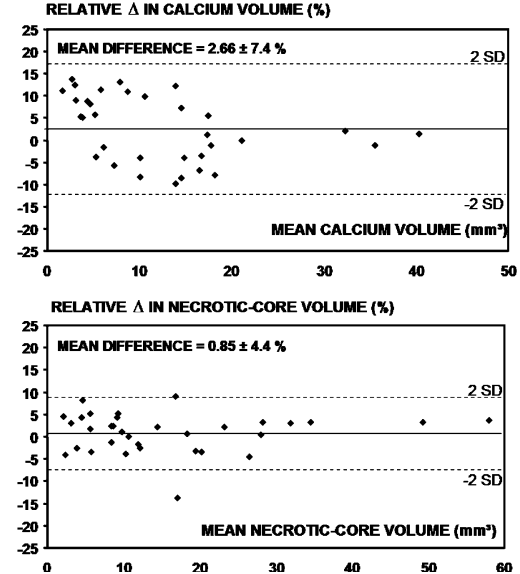

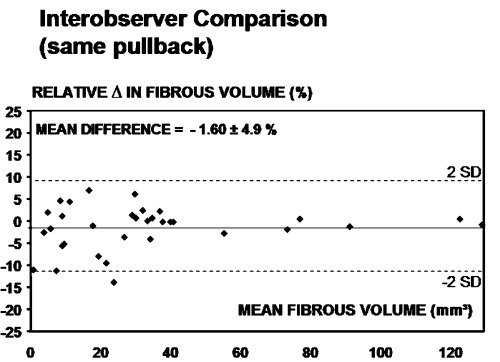

RELATIVE $\triangle$ IN FIBRO-LIPIDIC VOLUME (\%)

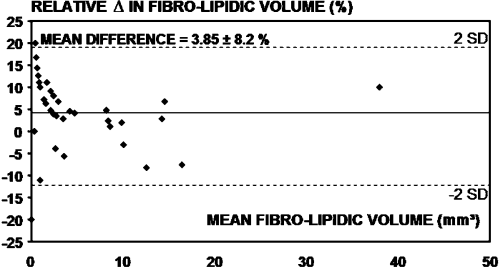

25 RELATIVE $\triangle$ IN CALCIUM VOLUME (\%)

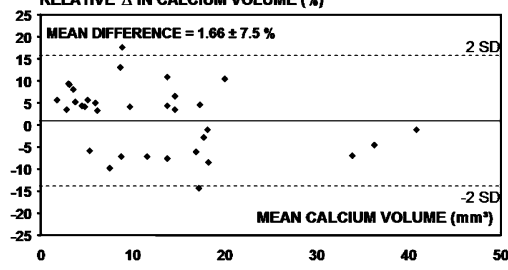

RELATIVE $\triangle$ IN NECROTIC-CORE VOLUME (\%)

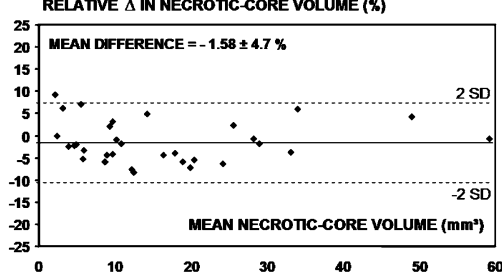

Fig. 3 Agreement of repeated VH-IVUS measurements of compositional volumes. Bland-Altman plots of intra- (left, mid) and interobserver (right) comparisons. $\Delta=$ difference

necrotic-core area without evident overlying fibrous tissue and a plaque burden area of $\geq 40 \%$ [18]. Because of the two-dimensional nature of the definition of $R F$ IVUS derived TCFA, serial assessment may be difficult as outlined above; this may result in a higher measurement variability. Besides, if the luminal border is shared by thrombus-which is more likely in coronary instability - the measurement variability of plaque and lumen dimensions may be even higher, as thrombus may be misclassified as fibrous tissue [35].

A volumetric parameter that is less dependent on matching problems is the RF-IVUS derived necroticcore volume. From a pathophysiological point of view the necrotic core volume is a very interesting target, as both the necrotic core size and its longitudinal extent are related to the risk of plaque rupture that may trigger cardiovascular events as demonstrated by histopathological studies [4, 5, 36, 37]. In agreement with these findings, Hong et al. recently found a significantly higher RF-IVUS derived necrotic-core volume in lesions of patients with acute coronary syndromes compared to patients with stable angina [22]. In our study in patients with stable angina, necrotic-core volume showed on average the highest reproducibility of all plaque components. Based on pathophysiological considerations and the favorable measurement reproducibility, the parameter RF-IVUS derived necrotic-core volume has the potential to become a major imaging target of future pharmacological intervention trials with IVUS. Nevertheless, the relation between serial changes of this parameter and clinical endpoints has to be demonstrated. 


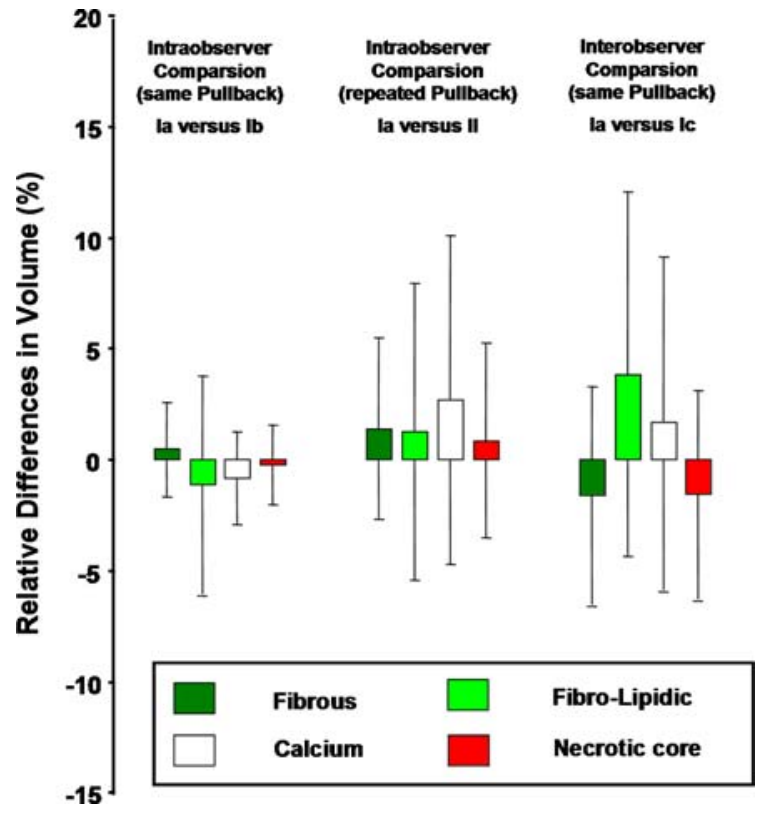

Fig. 4 Relative differences of repeated compositional VHIVUS volume measurements. The volumetric VH-IVUS measurements of plaque composition in intra- (left and mid bars) and interobserver comparisons (right bars) are displayed (mean $\pm 1 \mathrm{SD}$ ). The paired t-test Ia versus Ib; Ia versus II; and Ia versus Ic showed no significant difference ( $P=$ NS for all). The necrotic-core volume showed on average the lowest measurement variability

\section{Limitations}

To the best of our knowledge, this was the first study that tested the reproducibility of volumetric RFIVUS. The number of coronary segments was limited but comparable to other studies that addressed the reproducibility of IVUS measurements [13-15, 34, 38, 39]. All patients had stable angina pectoris. Similar to other studies with IVUS and RF-IVUS, we excluded tortuous and severely calcified vessels which could have led to non-uniform pullbacks [34]. We analyzed high quality RF-IVUS image runs with limited circumferential calcification which resulted in a relatively high accuracy of total vessel border detection [11, 12]. Therefore, measurement reproducibility may be somewhat lower in routine clinical IVUS cases; nevertheless, the selection criteria of target segments in serial IVUS studies are similar [7-10]. Inter-catheter and inter-system variability was not addressed [34, 38, 39]. The high accuracy of the off-line RF-IVUS analysis was achieved at the expense of manual correction of the automatically derived lumen and vessel contours; accordingly, our data may not be generalized to online application of RF-IVUS.

\section{Conclusions}

In mild-to-moderate atherosclerotic coronary artery disease, the reproducibility of volumetric compositional RF-IVUS measurements from the same pullback is relatively high, but lower than the reproducibility of geometrical IVUS measurements. Measurements from repeated pullbacks and by different observers show acceptable reproducibilities for the use in a serial study design; the volumetric measurement of the necroticcore shows on average the highest reproducibility of the compositional RF-IVUS measurements.

\section{References}

1. Waters D, Craven TE, Lesperance J (1993) Prognostic significance of progression of coronary atherosclerosis. Circulation 87:1399-1401

2. Ambrose JA, Tannenbaum MA, Alexopoulos D, Hjemdahl-Monsen CE, Leavy J, Weiss M et al (1988) Angiographic progression of coronary artery disease and the development of myocardial infarction. J Am Coll Cardiol 12:56-62

3. Shah PK (2003) Mechanisms of plaque vulnerability and rupture. J Am Coll Cardiol 41:15-22. doi:10.1016/S07351097(02)02834-6

4. Burke AP, Farb A, Malcom GT, Liang YH, Smialek J, Virmani R (1997) Coronary risk factors and plaque morphology in men with coronary disease who died suddenly. N Engl J Med 336:1276-1282. doi:10.1056/NEJM199705 013361802

5. Kolodgie FD, Virmani R, Burke AP, Farb A, Weber DK, Kutys R et al (2004) Pathologic assessment of the vulnerable human coronary plaque. Heart 90:1385-1391. doi: 10.1136/hrt.2004.041798

6. Böse D, von Birgelen C, Erbel R (2007) Intravascular ultrasound for the evaluation of therapies targeting coronary atherosclerosis. J Am Coll Cardiol 49:925-932. doi: 10.1016/j.jacc.2006.08.067

7. Schartl M, Bocksch W, Koschyk DH, Voelker W, Karsch KR, Kreuzer J et al (2001) Use of intravascular ultrasound to compare effects of different strategies of lipid-lowering therapy on plaque volume and composition in patients with coronary artery disease. Circulation 104:387-392. doi: 10.1161/hc2901.093188

8. Jensen LO, Thayssen P, Pedersen KE, Stender S, Haghfelt $\mathrm{T}$ (2004) Regression of coronary atherosclerosis by simvastatin: a serial intravascular ultrasound study. Circulation 110:265-270. doi:10.1161/01.CIR.0000135215.75876.41 
9. Nissen SE, Nicholls SJ, Sipahi I, Libby P, Raichlen JS, Ballantyne CM et al (2006) Effect of very high-intensity statin therapy on regression of coronary atherosclerosis: the ASTEROID trial. JAMA 295:1556-1565. doi:10.1001/ jama.295.13.jpc60002

10. Tardif JC, Grégoire J, L'Allier PL, Ibrahim R, Lespérance J, Heinonen TM et al (2007) Effect of rHDL on atherosclerosis-safety and efficacy (ERASE) investigators. Effects of reconstituted high-density lipoprotein infusions on coronary atherosclerosis: a randomized controlled trial. JAMA 297:1675-1682. doi:10.1001/jama.297.15.jpc70004

11. Mintz GS, Nissen SE, Anderson WD, Bailey SR, Erbel R, Fitzgerald PJ et al (2001) American College of Cardiology clinical expert consensus document on standards for acquisition, measurement and reporting of intravascular ultrasound studies (IVUS). J Am Coll Cardiol 37:14781492. doi:10.1016/S0735-1097(01)01175-5

12. Di Mario C, Görge G, Peters R, Kearney P, Pinto F, Hausmann D et al (1998) Clinical application and image interpretation in intracoronary ultrasound. Study group on intracoronary imaging of the working group of coronary circulation and of the subgroup on intravascular ultrasound of the working group of echocardiography of the European society of cardiology. Eur Heart J 19:207-229. doi: 10.1053/euhj.1996.0433

13. von Birgelen C, de Vrey EA, Mintz GS, Nicosia A, Bruining $\mathrm{N}$, Li W et al (1997) ECG-gated three-dimensional intravascular ultrasound: feasibility and reproducibility of the automated analysis of coronary lumen and atherosclerotic plaque dimensions in humans. Circulation 96:2944-2952

14. Bruining N, von Birgelen C, de Feyter PJ, Ligthart J, Li W, Serruys PW et al (1998) ECG-gated versus nongated threedimensional intracoronary ultrasound analysis: implications for volumetric measurements. Catheter Cardiovasc Diagn 43:254-260. doi :10.1002/(SICI)1097-0304(199803) 43:3<254::AID-CCD3>3.0.CO;2-8

15. Jensen LO, Thayssen $P$ (2007) Accuracy of electrocardiographic-gated versus nongated volumetric intravascular ultrasound measurements of coronary arterial narrowing. Am J Cardiol 99:279-283. doi:10.1016/j.amjcard.2006.08.024

16. König A, Klauss V (2007) Virtual histology. Heart 93: 977-982. doi:10.1136/hrt.2007.116384

17. Mehta SK, McCrary JR, Frutkin AD, Dolla WJ, Marso SP (2007) Intravascular ultrasound radiofrequency analysis of coronary atherosclerosis: an emerging technology for assessment of vulnerable plaque. Eur Heart J 28:12831288. doi:10.1093/eurheartj/ehm112

18. Rodriguez-Granillo GA, García-García HM, McFadden EP, Valgimigli M, Aoki J, de Feyter P et al (2005) In vivo intravascular ultrasound-derived thin-cap fibroatheroma detection using ultrasound radiofrequency data analysis. J Am Coll Cardiol 46:2038-2042. doi:10.1016/j.jacc.2005. 07.064

19. Rodriguez-Granillo GA, García-García HM, Valgimigli M, Vaina S, van Mieghem C, van Geuns RJ et al (2006) Global characterization of coronary plaque rupture phenotype using three-vessel intravascular ultrasound radiofrequency data analysis. Eur Heart J 27:1921-1927. doi:10.1093/eurheartj/ ehl104

20. Valgimigli M, Rodriguez-Granillo GA, Garcia-Garcia HM, Malagutti P, Regar E, de Jaegere P et al (2006) Distance from the ostium as an independent determinant of coronary plaque composition in vivo: an intravascular ultrasound study based radiofrequency data analysis in humans. Eur Heart J 27:655-663. doi:10.1093/eurheartj/ehi716

21. Fujii K, Carlier SG, Mintz GS, Wijns W, Colombo A, Böse D et al (2005) Association of plaque characterization by intravascular ultrasound virtual histology and arterial remodeling. Am J Cardiol 96:1476-1483. doi:10.1016/ j.amjcard.2005.07.054

22. Hong MK, Mintz GS, Lee CW, Suh J, Kim JH, Park DW et al (2007) Comparison of virtual histology to intravascular ultrasound of culprit coronary lesions in acute coronary syndrome and target coronary lesions in stable angina pectoris. Am J Cardiol 100:953-959. doi:10.1016/j.amjcard.2007.04.034

23. Nair A, Margolis MP, Kuban BD, Vince DG (2007) Automated coronary plaque characterisation with intravascular ultrasound backscatter: ex vitro validation. Eurointervention 3:113-120

24. Nair A, Kuban BD, Tuzcu EM, Schoenhagen P, Nissen SE, Vince DG (2002) Coronary plaque classification with intravascular ultrasound radiofrequency data analysis. Circulation 106:2200-2206. doi:10.1161/01.CIR.0000035654.18341.5E

25. Nasu K, Tsuchikane E, Katoh O, Vince DG, Virmani R, Surmely JF et al (2006) Accuracy of in vivo coronary plaque morphology assessment: a validation study of in vivo virtual histology compared with in vitro histopathology. J Am Coll Cardiol 47:2405-2412. doi:10.1016/j.jacc.2006.02.044

26. Kawasaki M, Sano K, Okubo M, Yokoyama H, Ito Y, Murata I et al (2005) Volumetric quantitative analysis of tissue characteristics of coronary plaques after statin therapy using three-dimensional integrated backscatter intravascular ultrasound. J Am Coll Cardiol 45:1946-1953. doi:10.1016/j. jacc.2004.09.081

27. von Birgelen C, de Feyter PJ, de Vrey EA, Li W, Bruining N, Nicosia A et al (1997) Simpson's rule for the volumetric ultrasound assessment of atherosclerotic coronary arteries: a study with ECG-gated three-dimensional intravascular ultrasound. Coron Artery Dis 8:363-369. doi:10.1097/ 00019501-199706000-00005

28. Bland JM, Altman DG (1986) Statistical methods for assessing agreement between two methods of clinical measurement. Lancet 2:307-310

29. Hartmann M, von Birgelen C, Mintz GS, Stoel MG, Eggebrecht H, Wieneke H et al (2006) Relation between lipoprotein(a) and fibrinogen and serial intravascular ultrasound plaque progression in left main coronary arteries. J Am Coll Cardiol 48:446-452. doi:10.1016/j.jacc.2006.03.047

30. von Birgelen C, Hartmann M, Mintz GS, Baumgart D, Schmermund A, Erbel R (2003) Relation between progression and regression of atherosclerotic left main coronary artery disease and serum cholesterol levels as assessed with serial long-term ( $\geq 12$ months) follow-up intravascular ultrasound. Circulation 108:2757-2762. doi: 10.1161/01.CIR.0000103664.47406.49

31. von Birgelen C, Hartmann M, Mintz GS, van Houwelingen KG, Deppermann N, Schmermund A et al (2004) Relationship between cardiovascular risk as predicted by established risk scores versus plaque progression as measured by serial intravascular ultrasound in left main coronary arteries. Circulation 110:1579-1585. doi:10.1161/01.CIR.0000142048. 94084.CA 
32. Ambrose JA, D'Agate DJ (2005) Classification of systemic therapies for potential stabilization of the vulnerable plaque to prevent acute myocardial infarction. Am J Cardiol 95:379-382. doi:10.1016/j.amjcard.2004.09.037

33. http://www.clinicaltrials.gov/ct/show/NCT00268996. November 2007

34. Rodriguez-Granillo GA, Vaina S, García-García HM, Valgimigli M, Duckers E, van Geuns RJ et al (2006) Reproducibility of intravascular ultrasound radiofrequency data analysis: implications for the design of longitudinal studies. Int J Cardiovasc Imaging 22:621-631. doi:10.1007/ s10554-006-9080-0

35. Frutkin AD, Metha SK, McCrary JR, Marso SP (2007) Limitations to the use of virtual histology-intravascular ultrasound to detect vulnerable plaque. Eur Heart J 28:1783-1784. doi:10.1093/eurheartj/ehm145

36. Virmani R, Burke AP, Farb A, Kolodgie FD (2006) Pathology of the vulnerable plaque. J Am Coll Cardiol 47:C13-C18. doi:10.1016/j.jacc.2005.10.065
37. Burke AP, Virmani R, Galis Z, Haudenschild CC, Muller JE (2003) 34th Bethesda conference: task force\#2-what is the pathologic basis for new atherosclerosis imaging techniques? J Am Coll Cardiol 41:1874-1886. doi: 10.1016/S0735-1097(03)00359-0

38. Hartmann M, von Birgelen C, Mintz GS, Deppermann N, Dirsch O, Stoel MG et al (2006) Dedicated calibration formulas permit correction of differences between measurements by different IVUS devices as demonstrated in atherosclerotic human coronary arteries in vitro. Int $\mathrm{J}$ Cardiovasc Imaging 22:605-613. doi:10.1007/s10554-0069092-9

39. Rodriguez-Granillo GA, Bruining N, McFadden E, Ligthart JM, Aoki J, Regar E et al (2005) Geometrical validation of intravascular ultrasound radiofrequency data analysis (virtual histology) acquired with a $30 \mathrm{MHz}$ boston scientific corporation imaging catheter. Catheter Cardiovasc Interv 66:514-518. doi:10.1002/ccd.20447 